\title{
Lepton Flavour Violation in Tau Decays: Results and Prospects at the LHC
}

\author{
Kristof De Bruyn on behalf of the ATLAS, CMS and LHCb collaborations \\ Centre de Physique des Particules de Marseille (CPPM), 163 Avenue de Luminy, 13009 Marseille, France
}

\begin{abstract}
In this report, searches for the lepton flavour violating decays $\tau^{-} \rightarrow \mu^{-} \mu^{+} \mu^{-}, Z^{0} \rightarrow \tau^{ \pm} \mu^{\mp}$ and $D^{0} \rightarrow e^{ \pm} \mu^{\mp}$ are presented, using proton-proton collision data recorded by the LHC experiments during 2011 and 2012. The ATLAS experiment puts an upper limit on $\mathcal{B}\left(\tau^{-} \rightarrow \mu^{-} \mu^{+} \mu^{-}\right)<3.76 \times 10^{-7}$ at $90 \%$ confidence level, and on $\mathcal{B}\left(Z^{0} \rightarrow \tau^{ \pm} \mu^{\mp}\right)<$ $1.69 \times 10^{-5}$ at $95 \%$ confidence level, based on a data sample corresponding to an integrated luminosity of $20.3 \mathrm{fb}^{-1}$. The LHCb experiment puts an upper limit on $\mathcal{B}\left(\tau^{-} \rightarrow \mu^{-} \mu^{+} \mu^{-}\right)<4.6 \times 10^{-8}$ and on $\mathcal{B}\left(D^{0} \rightarrow e^{ \pm} \mu^{\mp}\right)<1.3 \times 10^{-8}$, both at $90 \%$ confidence level, based on a data sample corresponding to an integrated luminosity of $3.0 \mathrm{fb}^{-1}$.
\end{abstract}

Keywords: Lepton flavour violation, Rare decays

\section{Introduction}

In the Standard Model (SM), lepton flavour violation (LFV) involving charged leptons is induced by oscillations between the three neutrino flavour eigenstates. LFV decays can therefore only proceed through loop processes, which are suppressed by a factor $\left(m_{v} / m_{W}\right)^{4}$. The resulting SM branching fractions are of the order of $10^{-40}$ or smaller, out of reach for current and future experimental measurements. This makes LFV tests in the charged lepton sector an ideal playground to search for beyond the SM physics, and in particular for those models that introduce new particles around the $\mathrm{TeV}$ scale, aiming for a discovery at the LHC. These theories often have strongly enhanced rates for LFV decays, providing the opportunity to observe them with current experiments.

This report presents three searches for LFV decays at the LHC: A search for $\tau^{-} \rightarrow \mu^{-} \mu^{+} \mu^{-}$, performed by the ATLAS [1] and LHCb [2] experiments, for $Z^{0} \rightarrow \tau^{ \pm} \mu^{\mp}$, performed by ATLAS [3], and for $D^{0} \rightarrow e^{ \pm} \mu^{\mp}$, performed by LHCb [4]. The ATLAS searches use protonproton collision data corresponding to an integrated luminosity of $20.3 \mathrm{fb}^{-1}$ collected by the ATLAS detector in 2012 at a centre-of-mass energy of $8 \mathrm{TeV}$. The ATLAS detector, described in detail in Ref. [5], is a multi- purpose particle detector with a forward-backward symmetric cylindrical geometry and a near $4 \pi$ coverage in solid angle. The LHCb searches, on the other hand, use a data sample corresponding to an integrated luminosity of $3.0 \mathrm{fb}^{-1}$ collected by the $\mathrm{LHCb}$ detector in 2011 and 2012 at centre-of-mass energies of 7 and $8 \mathrm{TeV}$, respectively. The LHCb detector, described in detail in Refs. $[6,7]$, is a single-arm forward spectrometer covering the pseudo-rapidity range $2<\eta<5$, designed for the study of particles containing $b$ or $c$ quarks.

\section{Limit on $\mathcal{B}\left(\tau^{-} \rightarrow \mu^{-} \mu^{+} \mu^{-}\right)$from ATLAS}

The ATLAS experiment has performed a search for the neutrinoless decay $\tau^{-} \rightarrow \mu^{-} \mu^{+} \mu^{-}$using a sample of $W^{-} \rightarrow \tau^{-} \bar{v}_{\tau}$ decays. This production channel is preferred over other options, like $Z^{0} \rightarrow \tau^{+} \tau^{-}$or decays of heavy flavour, as it has the highest trigger and reconstruction efficiencies. These efficiencies are limited by the relatively low transverse momentum, $p_{\mathrm{T}}$, of the final-state muons compared to other objects used by the ATLAS trigger and event reconstruction systems. The contamination to the selected data sample from other tau production sources is estimated to be less than $3 \%$.

The signal yield $N_{\text {sig }}$ passing the below described selection requirements is translated into a branching frac- 
tion by comparing it to the measured $W^{-} \rightarrow \tau^{-} \bar{v}_{\tau}$ cross section $\sigma_{W^{-} \rightarrow \tau^{-} \bar{v}_{\tau}}[8]$ as

$$
\mathcal{B}\left(\tau^{-} \rightarrow \mu^{-} \mu^{+} \mu^{-}\right)=\frac{N_{\text {sig }}}{\epsilon_{\text {sig }} \times \sigma_{W^{-} \rightarrow \tau^{-} \bar{v}_{\tau}} \times \mathcal{L}},
$$

where $\epsilon_{\text {sig }}$ is the signal selection efficiency and $\mathcal{L}$ is the integrated luminosity of the data sample. Using the results from Ref. [8] leads to a normalisation factor $\sigma \times \mathcal{L}=(2.41 \pm 0.08) \times 10^{8}$.

The $\tau^{-} \rightarrow \mu^{-} \mu^{+} \mu^{-}$candidates are selected in three steps. First, a loose cut-based selection, aimed at rejecting background that is kinematically inconsistent with the signal, is performed to obtain a background sample that can be used to train a boosted decision tree (BDT). Next, additional "tight" selection requirements are imposed, including an initial loose cut $x_{0}$ on the output $x$ of the BDT. Finally, the cut $x_{1}>x_{0}$ on the BDT output is optimised for the expected branching fraction limit. The final selection efficiency is $\epsilon_{\text {sig }}=$ $0.0231 \pm 0.0005$ (Jet) $\pm 0.0009(\mathrm{MC}) \pm 0.0025$ (trig) \pm 0.0030 (reco) for signal candidates in the search window $m_{3 \mu} \in[1713,1841] \mathrm{MeV} / c^{2}$, defined using the threemuon invariant mass.

The expected background yield in the signal search window after the "tight $+x>x_{1}$ " selection is calculated using the following procedure: For the "tight $+x>x_{0}$ " selection, the background yield in the blinded signal region is estimated from a fit to the mass sidebands, as shown in Fig. 1. The expected yield is combined with the results from a fit to the BDT output in the region $x>x_{0}$ in order to extrapolate the background yield to the "tight $+x>x_{1}$ " selection. This leads to an expected background yield of $N_{\mathrm{bkg}}=0.193 \pm 0.037$ (stat) \pm 0.131 (syst), where the dominant (systematic) uncertainty is associated with the extrapolation procedure.

After unblinding, no events in the signal search window pass the final selection requirements. An upper limit [1]

$$
\mathcal{B}\left(\tau^{-} \rightarrow \mu^{-} \mu^{+} \mu^{-}\right)<3.76 \times 10^{-7} @ 90 \% \text { C.L. }
$$

is set on the $\tau^{-} \rightarrow \mu^{-} \mu^{+} \mu^{-}$branching fraction using the CLs method $[9,10]$. Although this limit is not yet competitive with the results from the LHCb [2] and Belle [11] experiments, it demonstrates ATLAS' potential for performing competitive LFV searches using the LHC run 2 data and beyond, and its capabilities to reconstruct and trigger on low- $p_{\mathrm{T}}$ muons.

\section{Limit on $\mathcal{B}\left(\tau^{-} \rightarrow \mu^{-} \mu^{+} \mu^{-}\right)$from $\mathrm{LHCb}$}

The LHCb experiment has performed a search for the neutrinoless decay $\tau^{-} \rightarrow \mu^{-} \mu^{+} \mu^{-}$using a sample of tau

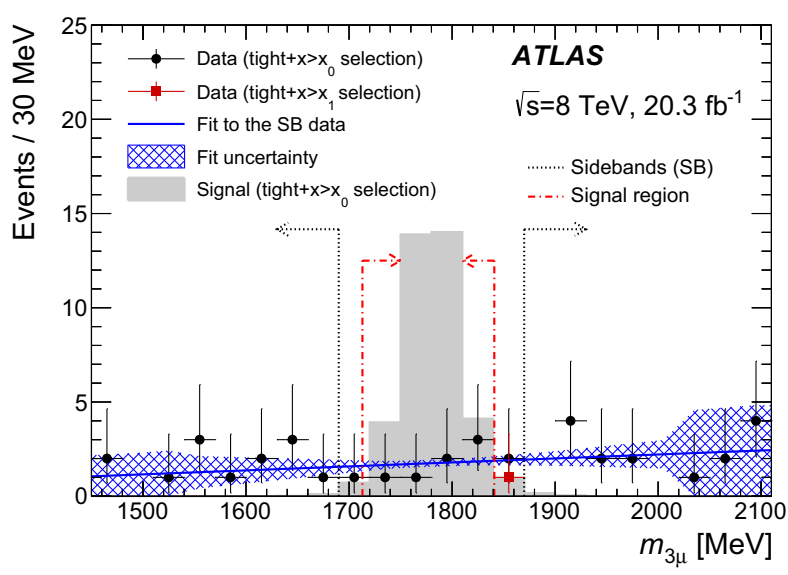

Figure 1: Three-muon invariant mass distribution of the ATLAS search for $\tau^{-} \rightarrow \mu^{-} \mu^{+} \mu^{-}$. The circles show the data after the tight $+x>x_{0}$ selection, and the square shows the single data point remaining after the tight $+x>x_{1}$ selection. The sideband and signal regions are indicated by the dotted and dash-dotted arrows, respectively.

leptons from $b$ and $c$-hadron decays. The signal yield is translated into a branching fraction by comparing it to the yield observed in the normalisation mode $D_{s}^{-} \rightarrow$ $\phi\left(\rightarrow \mu^{+} \mu^{-}\right) \pi^{-}$as

$$
\begin{array}{r}
\mathcal{B}\left(\tau^{-} \rightarrow \mu^{-} \mu^{+} \mu^{-}\right)=\frac{\mathcal{B}\left(D_{s}^{-} \rightarrow \phi\left(\rightarrow \mu^{+} \mu^{-}\right) \pi^{-}\right)}{\mathcal{B}\left(D_{s}^{-} \rightarrow \tau^{-} \bar{v}_{\tau}\right)} \\
\times f_{\tau}^{D_{s}} \times \frac{\epsilon_{\text {norm }}}{\epsilon_{\text {sig }}} \times \frac{N_{\text {sig }}}{N_{\text {cal }}}=\alpha \times N_{\text {sig }} .
\end{array}
$$

Here, $f_{\tau}^{D_{s}}=0.80 \pm 0.03$ is the fraction of tau leptons that originates from a $D_{s}$ meson, calculated using the measured $b \bar{b}$ and $c \bar{c}$ cross-sections [12] and inclusive $b \rightarrow D_{s}, c \rightarrow D_{s}, b \rightarrow \tau$ and $c \rightarrow \tau$ branching fractions [13]. This leads to normalisation factors $\alpha(7 \mathrm{TeV})=$ $(7.20 \pm 0.98) \times 10^{-9}$ and $\alpha(8 \mathrm{TeV})=(3.37 \pm 0.50) \times 10^{-9}$, where the difference is due to changes in the trigger settings between 2011 and 2012 .

After an initial cut-based selection, the $\tau^{-} \rightarrow \mu^{-} \mu^{+} \mu^{-}$ candidates are identified using three likelihood variables: a multivariate classifier $\mathcal{M}_{3 \text { body }}$ that exploits the geometrical and kinematic properties of the $\tau^{-} \rightarrow$ $\mu^{-} \mu^{+} \mu^{-}$decay, the response $\mathcal{M}_{\mathrm{PID}}$ of a neural network giving the likelihood that each of the three final-state particles is compatible with the muon hypothesis, and the three-muon invariant mass $m_{3 \mu}$, whose distribution is shown in Fig. 2. The $\mathcal{M}_{3 \text { body }}$ and $\mathcal{M}_{\text {PID }}$ classifiers are trained on simulated signal events, and afterwards corrected for data-simulation differences using the $D_{s}^{-} \rightarrow$ $\phi\left(\rightarrow \mu^{+} \mu^{-}\right) \pi^{-}$normalisation mode and $J / \psi \rightarrow \mu^{+} \mu^{-}$decays in data, respectively. 


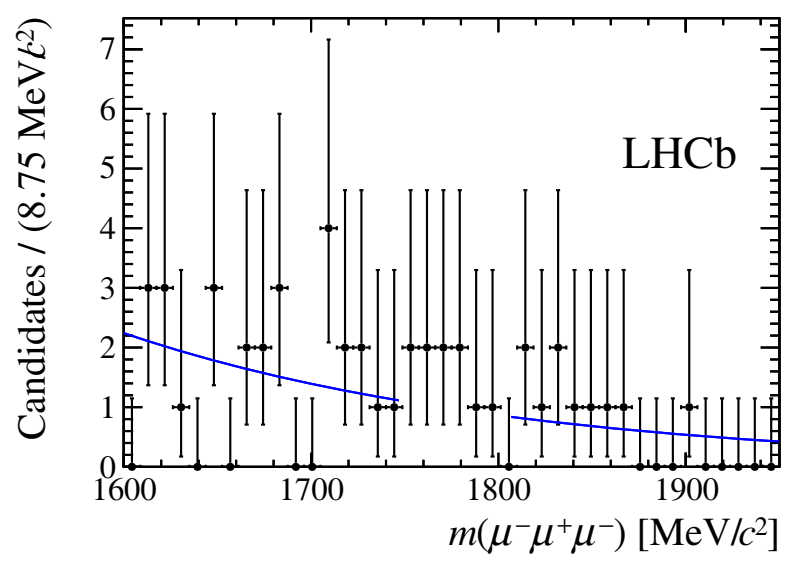

Figure 2: Three-muon invariant mass distribution and accompanying fit to the mass sidebands in the $\mathcal{M}_{3 \text { body }} \times \mathcal{M}_{\text {PID }}$ bin that contains the highest signal probability for the LHCb search for $\tau^{-} \rightarrow \mu^{-} \mu^{+} \mu^{-}$.

A combined fit is performed to the three-muon invariant mass distribution of the most sensitive bins in $\mathcal{M}_{3 \text { body }} \times \mathcal{M}_{\text {PID }}$. The result is consistent with the background-only hypothesis, and an upper limit [2]

$$
\mathcal{B}\left(\tau^{-} \rightarrow \mu^{-} \mu^{+} \mu^{-}\right)<4.6 \times 10^{-8} @ 90 \% \text { C.L. }
$$

is set on the branching fraction using the CLs method $[9,10]$. This limit is a factor 2.5 higher than the current best limit [11], set by the Belle experiment.

\section{Limit on $\mathcal{B}\left(Z^{0} \rightarrow \tau^{ \pm} \mu^{\mp}\right)$ from ATLAS}

The ATLAS experiment has performed a search for the LFV decay $Z^{0} \rightarrow \tau^{ \pm} \mu^{\mp}$, where the tau lepton is reconstructed in the three-pion final state. The analysis strategy is similar to the search for the LFV decay $H \rightarrow \mu^{ \pm} \tau^{\mp}[14,3]$, and both studies are performed in tandem. The selection requirements are optimised separately to account for the difference in kinematics of the $Z^{0}$ and Higgs boson decay products.

The main sources of background for this search are $W+$ jets, $Z^{0} \rightarrow \tau^{+} \tau^{-}$decays and combinatorial background. The combinatorial background is described using the same-sign $\tau^{ \pm} \mu^{ \pm}$candidates in the data. The $Z^{0} \rightarrow \tau^{+} \tau^{-}$background is modelled with an embedding technique: In a sample of $Z^{0} \rightarrow \mu^{+} \mu^{-}$events from data, the two muon tracks associated with the signal candidate are replaced by simulated $\tau^{-} \rightarrow \pi^{-} \pi^{+} \pi^{-} v_{\tau}$ decays. Finally, the $W+$ jets background is modelled using a control region in the data. This region is defined using the transverse mass

$$
m_{\mathrm{T}}^{\ell, E_{\mathrm{T}}^{\mathrm{miss}}} \equiv \sqrt{2 p_{\mathrm{T}}^{\ell} E_{\mathrm{T}}^{\mathrm{miss}}(1-\cos \Delta \phi)}
$$

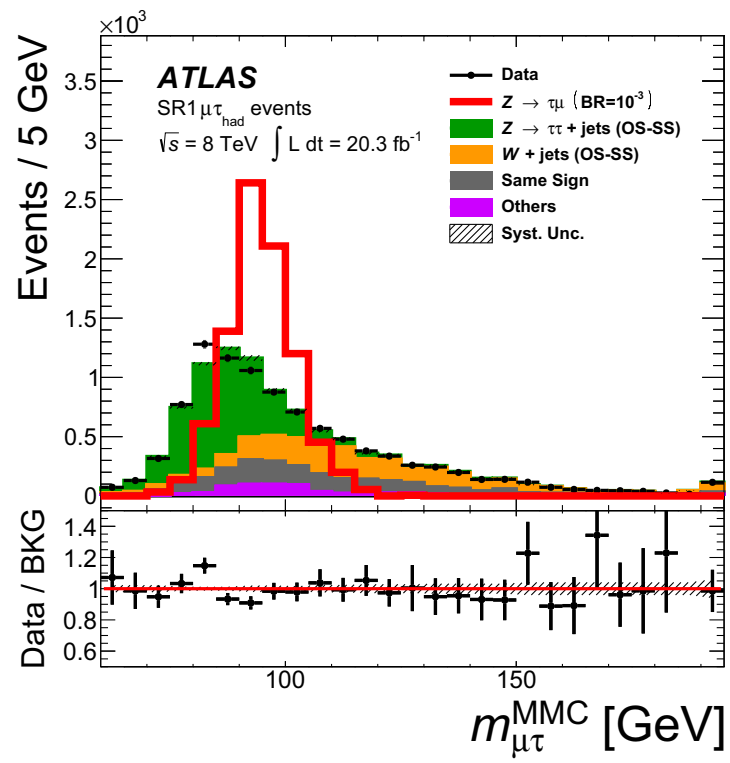

Figure 3: The invariant mass distribution, reconstructed from the observed tau, muon and missing transverse energy, for signal region 1 of the ATLAS search for $Z^{0} \rightarrow \tau^{ \pm} \mu^{\mp}$. The empty histogram gives an illustration of a potential signal distribution assuming a branching fraction of $10^{-3}$.

of the tau and muon candidates, where $\Delta \phi$ is the angle between the lepton $\ell$ and the direction of the missing transverse energy $E_{\mathrm{T}}^{\text {miss }}$. For the $W+$ jets control region $m_{\mathrm{T}}^{\mu, E_{\mathrm{T}}^{\text {miss }}}>60 \mathrm{GeV}$ and $m_{\mathrm{T}}^{\tau, E_{\mathrm{T}}^{\text {miss }}}>40 \mathrm{GeV}$.

Two signal search regions are identified using the transverse mass variables. For the first region $30<$ $m_{\mathrm{T}}^{\mu, E_{\mathrm{T}}^{\text {miss }}}<75 \mathrm{GeV}$ and $m_{\mathrm{T}}^{\tau, E_{\mathrm{T}}^{\text {miss }}}<20 \mathrm{GeV}$, while for the second region $m_{\mathrm{T}}^{\mu, E_{\mathrm{T}}^{\text {miss }}}<30 \mathrm{GeV}$ and $m_{\mathrm{T}}^{\tau, E_{\mathrm{T}}^{\text {miss }}}<45 \mathrm{GeV}$. A simultaneous fit of the invariant mass distribution, reconstructed from the observed tau, muon and missing transverse energy, is performed to the two signal and $W+$ jets control regions. A slight deficit in the number of observed events compared to the predicted background is found. For signal region 1, the result of this fit is shown in Fig. 3, together with an illustration of a potential signal distribution. The result is consistent with the background-only hypothesis, and an upper limit [3]

$$
\mathcal{B}\left(Z^{0} \rightarrow \tau^{ \pm} \mu^{\mp}\right)<1.69 \times 10^{-5} @ 95 \% \text { C.L. }
$$

is set on the branching fraction using the CLs method $[9,10]$. 


\section{Limit on $\mathcal{B}\left(D^{0} \rightarrow e^{ \pm} \mu^{\mp}\right)$ from $\mathrm{LHCb}$}

The $\mathrm{LHCb}$ experiment has performed a search for the LFV decay $D^{0} \rightarrow e^{ \pm} \mu^{\mp}$. Candidate $D^{0}$ mesons are selected from a sample of $D^{*+} \rightarrow D^{0} \pi^{+}$decays. The signal yield is translated into a branching fraction by comparing it to the yield observed in the normalisation mode $D^{0} \rightarrow K^{-} \pi^{+}$as

$$
\mathcal{B}\left(D^{0} \rightarrow e^{ \pm} \mu^{\mp}\right)=\mathcal{B}\left(D^{0} \rightarrow K^{-} \pi^{+}\right) \times \frac{\epsilon_{\text {norm }}}{\epsilon_{\text {sig }}} \times \frac{N_{\text {sig }}}{N_{\text {norm }}} .
$$

The selection efficiencies for the signal and normalisation channel are $\epsilon_{\text {sig }}=(4.4 \pm 0.3) \times 10^{-4}$ and $\epsilon_{\text {norm }}=$ $(2.5 \pm 0.1) \times 10^{-6}$, respectively. It should be noted that only $1 \%$ of the $D^{0} \rightarrow K^{-} \pi^{+}$candidates are retained by the trigger to cope with the large charm production rate at the LHC.

After an initial cut-based selection, a BDT is used to divide the sample of $D^{0} \rightarrow e^{ \pm} \mu^{\mp}$ candidates into three bins of different signal purity. Before imposing the selection requirements, the measured momentum of the electron candidates is corrected to account for loss of momentum by bremsstrahlung in the detector, using the photon energy deposition in the electromagnetic calorimeter.

In each of the three BDT bins, a two-dimensional fit is performed to the $D^{0}$ mass $m_{D^{0}}$ and $D^{*+}$ to $D^{0}$ mass difference $\Delta m=m_{D^{*+}}-m_{D^{0}}$. The result of this fit in the bin with the highest signal purity is shown in Fig. 4. The main source of background comes from $D^{0} \rightarrow \pi^{+} \pi^{-}$decays where one of the pions is misidentified as an electron and the other as a muon. The probability for this double misidentification to happen is only $(1.8 \pm 0.4) \times 10^{-8}$. But given the large $D^{0} \rightarrow \pi^{+} \pi^{-}$ branching fraction of $(1.421 \pm 0.025) \times 10^{-3}$ [13], a nonnegligible amount of $D^{0} \rightarrow \pi^{+} \pi^{-}$candidates still remain in the sample after the selection.

The fit is consistent with the background-only hypothesis. From a simultaneous fit to the three BDT bins a signal yield $N_{\text {sig }}=-7 \pm 15$ is obtained, and an upper limit [4]

$$
\mathcal{B}\left(D^{0} \rightarrow e^{ \pm} \mu^{\mp}\right)<1.3 \times 10^{-8} \quad @ 90 \% \text { C.L. }
$$

is set on the branching fraction using the CLs method [9, 10]. This result improves upon the previous best limit from the Belle experiment [15] by a factor 20 .

\section{References}

[1] G. Aad, et al., [ATLAS collaboration], Probing lepton flavour violation via neutrinoless $\tau^{-} \rightarrow \mu^{-} \mu^{+} \mu^{-}$decays with the ATLAS detector, Eur. Phys. J. C76 (5) (2016) 232. arXiv:1601.03567.

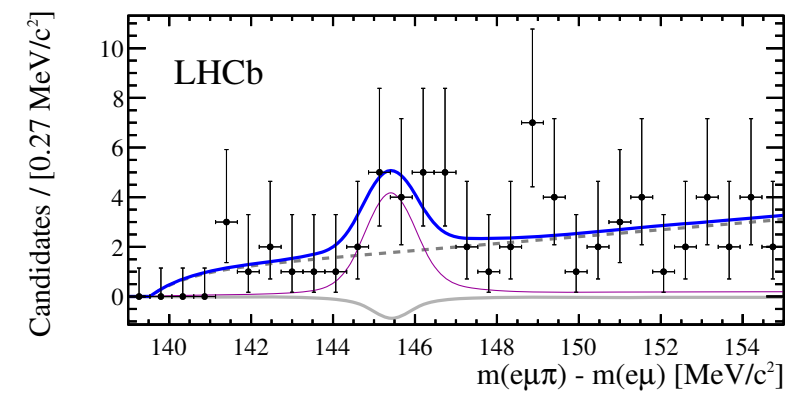

Figure 4: Distribution of the mass difference $m_{D^{*+}}-m_{D^{0}}$ in the bin that contains the highest signal probability for the $\mathrm{LHCb}$ search for $D^{0} \rightarrow e^{ \pm} \mu^{\mp}$. The solid top line shows the total fit, while the thick bottom line shows the $D^{0} \rightarrow e^{ \pm} \mu^{\mp}$ component, the thin line shows the misidentified $D^{0} \rightarrow \pi^{+} \pi^{-}$contribution and the dashed lines indicates the combinatorial background.

[2] R. Aaij, et al., [LHCb collaboration], Search for the lepton flavour violating decay $\tau^{-} \rightarrow \mu^{-} \mu^{+} \mu^{-}$, JHEP 02 (2015) 121. arXiv: 1409.8548.

[3] G. Aad, et al., [ATLAS collaboration], Search for leptonflavour-violating decays of the Higgs and $Z$ bosons with the ATLAS detectorarXiv:1604.07730.

[4] R. Aaij, et al., [LHCb collaboration], Search for the leptonflavour violating decay $D^{0} \rightarrow e^{ \pm} \mu^{\mp}$, Phys. Lett. B754 (2016) 167-175. arXiv: 1512.00322 .

[5] G. Aad, et al., [ATLAS collaboration], The ATLAS Experiment at the CERN Large Hadron Collider, JINST 3 (2008) S08003.

[6] A. A. Alves Jr., et al., [LHCb collaboration], The LHCb detector at the LHC, JINST 3 (2008) S08005.

[7] R. Aaij, et al., [LHCb collaboration], LHCb detector performance, Int. J. Mod. Phys. A30 (2015) 1530022. arXiv: 1412.6352.

[8] G. Aad, et al., [ATLAS collaboration], Measurement of the inclusive $W^{ \pm}$and $\mathrm{Z} /$ gamma cross sections in the electron and muon decay channels in $p p$ collisions at $\sqrt{s}=7 \mathrm{TeV}$ with the ATLAS detector, Phys. Rev. D85 (2012) 072004. arXiv: 1109.5141.

[9] A. L. Read, Presentation of search results: The CL(s) technique, J. Phys. G28 (2002) 2693-2704.

[10] G. Cowan, K. Cranmer, E. Gross, O. Vitells, Asymptotic formulae for likelihood-based tests of new physics, Eur. Phys. J. C71 (2011) 1554. arXiv:1007.1727.

[11] K. Hayasaka, et al., [Belle collaboration], Search for lepton flavor violating tau decays into three leptons with 719 million produced $\tau^{+} \tau^{-}$pairs, Phys. Lett. B687 (2010) 139-143. arXiv:1001.3221.

[12] R. Aaij, et al., [LHCb collaboration], Prompt charm production in pp collisions at sqrt(s)=7 TeV, Nucl. Phys. B871 (2013) 1-20. arXiv:1302.2864.

[13] C. Patrignani, [Particle Data Group], Review of Particle Physics, Chin. Phys. C40 (10) (2016) 100001.

[14] G. Aad, et al., [ATLAS collaboration], Search for leptonflavour-violating $H \rightarrow \mu \tau$ decays of the Higgs boson with the ATLAS detector, JHEP 11 (2015) 211. arXiv:1508.03372.

[15] M. Petric, et al., [Belle collaboration], Search for leptonic decays of $D^{0}$ mesons, Phys. Rev. D81 (2010) 091102. arXiv:1003.2345. 